\title{
CENTRIFUGAL DISTORTION EFFECT IN DIATOMIC MOLECULES DESCRIBED BY MORSE POTENTIAL*
}

\begin{abstract}
M. MoLSKI
Department of Theoretical Chemistry, Faculty of Chemistry

A. Mickiewicz University, Grunwaldzka 6, 60-780 Poznani, Poland

(Received November 12, 1992; revised version January 26, 1999)

An attempt at including the centrifugal distortion effect in description of the rovibrational states of diatomic systems with a molecular potential approximated by the Morse function is presented. The derived Schrödinger equation can be rigorously solved leading to the eigenvalues in the form of a continued fraction, which are applied for evaluation of the molecular constants and for prediction of the rovibrational spectra of the selected diatomic molecules giving quite satisfactory reproduction of the experimental data. A simple extension of the method developed, by assuming the rotational dependence of the dissociation constant, is also proposed.
\end{abstract}

PACS numbers: 33.10.Cs, 33.10.Jz

\section{Introduction}

The well resolved IR and MW spectra, as well as the structural simplicity of diatomic systems are the reasons why they have become convenient test objects for the new spectroscopic theories and molecular models.

Recently, on the basis of the deformable body model [1] and harmonic [2-5] or Simons-Parr-Finlan [6-10] potential approximation, a few different approaches to theoretical reproduction of the rovibrational spectra and calculation of molecular parameters have been proposed. A common feature of these methods is that they take into account the deformational influence of the centrifugal force acting in the rovibrational systems, and lead to the exact analytic formulae for the rovibrational energy and the corresponding wave functions.

In this paper we propose to extend the research area onto the diatomic molecules described by the Morse potential [11]:

$$
U(r)=D_{0}\left\{1-\exp \left[-a\left(r-r_{0}\right) / r_{0}\right]\right\}^{2},
$$

*This research was supported by the grant of the Committee for Scientific Research No. 2066391.01. 
in which $r$ and $r_{0}$ denote the internuclear separation and equilibrium distance, respectively, whereas $D_{0}$ represents the depth of the potential well and $a$ is the range parameter. The Morse potential has a few remarkable properties, making it useful for approximating the real potential curves of diatomic systems. Namely:

(i) It takes into account the anharmonicity of vibrational motions.

(ii) It includes the ability of the molecules to undergo dissociation at high excitation energies.

(iii) Its behaviour over the whole range of internuclear distance is qualitatively correct (including asymptotic condition $U(\infty)=D_{0}$ ).

(iv) It preserves the quasi-harmonic properties in the vicinity of the potential minimum.

(v) The rovibrational Schrödinger equation with potential (1) can be rigorously solved [12].

In view of the above the aim of this paper is to employ the deformable body model $[1,2]$ in description of rovibrational states of diatomic systems with a molecular potential approximated by the Morse function (1). The presented approach leads to the Schrödinger equation, providing analytic wave functions and eigenvalues given in the form of a continued fraction, which will be applied for evaluation of molecular constants and for prediction of rovibrational spectra of the selected diatomic molecules. We shall also be concerned with a modification of the obtained formula by taking advantage of the expansion of dissociation constant into a continued fraction of rotational quantum number.

\section{Method and applications}

The rovibrational Schrödinger equation for diatomic systems endowed with reduced mass $m$, and including the Morse function (1) has the form

$$
\begin{aligned}
& {\left[-\frac{\hbar^{2}}{2 m} \frac{\mathrm{d}^{2}}{\mathrm{~d} r^{2}}+\frac{\hbar^{2} J(J+1)}{2 m r^{2}}+D_{0}\left\{1-\exp \left[-a\left(r-r_{0}\right) / r_{0}\right]\right\}^{2}-E_{v J}\right]} \\
& \quad \times \psi_{v J}(r)=0 .
\end{aligned}
$$

Expanding the reciprocal moment of inertia in series of the curvilinear coordinate $z=\exp \left[-a\left(r-r_{0}\right) / r_{0}\right]$ [12], in the vicinity of the equilibrium configuration $r_{0}$, we get

$$
\begin{aligned}
& \frac{\hbar^{2} J(J+1)}{2 m r^{2}}=B J(J+1)\left(c_{0}+c_{1} z+c_{2} z^{2}+\ldots\right), \\
& B=\hbar^{2}\left(2 m r_{0}^{2}\right)^{-1}, \quad c_{0}=1-3 a^{-1}+3 a^{-2}, \\
& c_{1}=4 a^{-1}-6 a^{-2}, \quad c_{2}=-a^{-1}+3 a^{-2},
\end{aligned}
$$

whereas the original equation (2) for parabolic expansion takes the form

$$
\left(-\frac{\hbar^{2}}{2 m} \frac{\mathrm{d}^{2}}{\mathrm{~d} r^{2}}+f_{0}-2 f_{1} z+f_{2} z^{2}-E_{v J}\right) \psi_{v J}(r)=0
$$




$$
\begin{aligned}
& f_{0}=r_{0}^{2} a^{-2}\left[D_{0}+c_{0} B J(J+1)\right], \\
& f_{1}=r_{0}^{2} a^{-2}\left[D_{0}-c_{1} B J(J+1) / 2\right], \quad f_{2}=r_{0}^{2} a^{-2}\left[D_{0}+c_{2} B J(J+1)\right] .
\end{aligned}
$$

Taking into account a quantum-mechanical equation of motion in the Heisenberg representation, as well as the explicit form of Hamilton's operator occurring in (2), one gets a quantum-mechanical force acting in the rovibrational systems described by the Morse potential

$$
\widehat{\hat{p}}=(\mathrm{i} \hbar)^{-1}[\widehat{p}, \widehat{H}]=2 D_{0} r_{0} a \exp \left[-a\left(r-r_{0}\right) / r_{0}\right]\left\{1-\exp \left[-a\left(r-r_{0}\right) / r_{0}\right]\right\},
$$

or in an equivalent form

$$
\begin{aligned}
& \hat{\dot{p}}=2 D_{J} r_{J} a_{J} \exp \left[-a_{J}\left(r-r_{J}\right) / r_{J}\right]\left\{1-\exp \left[-a_{J}\left(r-r_{J}\right) / r_{J}\right]\right\}, \\
& r_{J}=r_{0}\left[1-a^{-1} \ln \left(f_{1} / f_{2}\right)\right], \quad a_{J}=a\left[1-a^{-1} \ln \left(f_{1} / f_{2}\right)\right], \\
& D_{J}=f_{2} \exp \left[-2 a\left(r_{J}-r_{0}\right) / r_{0}\right]=f_{1}^{2} / f_{2} .
\end{aligned}
$$

The quantum-mechanical force (10) is endowed with an effective potential

$$
U(r)_{\mathrm{eff}}=D_{J}\left\{1-\exp \left[-a_{J}\left(r-r_{J}\right) / r_{J}\right]\right\}^{2},
$$

which permits rewriting of (6) into an equation amenable to analytic solution

$$
\begin{aligned}
& \left(-\frac{\hbar^{2}}{2 m} \frac{\mathrm{d}^{2}}{\mathrm{~d} r^{2}}+D_{J}\left\{1-\exp \left[-a_{J}\left(r-r_{J}\right) / r_{J}\right]\right\}^{2}-\left(E_{v J}-E_{\text {eff }}^{\mathrm{Rot}}\right)\right) \\
& \quad \times \psi_{v J}(r)=0,
\end{aligned}
$$

where $r_{J}, D_{J}, a_{J}$ are the changed equilibrium configuration, the modified dissociation constant and the modified range parameter in the $J$-rotational state, whereas the last term $E_{\text {eff }}^{\text {Rot }}=f_{0}-f_{1}^{2} f_{2}^{-1}$ describes the effective rotational energy. The obtained results indicate that in rovibrational diatomic systems the time change of momentum occurs under the influence of force (10) including modified potential parameters $r_{J}, D_{J}$ and $a_{J}$; it is a consequence of the action of the centrifugal force which operates in all systems with rotational degrees of freedom. A look into (6) reveals that

$$
E_{\text {eff }}^{\text {Rot }}=f_{0}-2 f_{1} z_{J}+f_{2} z_{J}^{2}=f_{0}-f_{1}^{2} f_{2}^{-1}, \quad z_{J}=\exp \left[-a\left(r_{J}-r_{0}\right) / r_{0}\right],
$$

i.e., the effective rotation energy is a result of a change of the equilibrium configuration $r_{0} \rightarrow r_{J}$ caused by the centrifugal force. The Schrödinger equation (14) has the solutions $[11,12]$

$$
\begin{aligned}
& E_{v J}=f_{0}-f_{1}^{2} f_{2}^{-1}+\hbar \omega_{J}(v+1 / 2)-\hbar \omega_{J} x_{J}(v+1 / 2)^{2}, \quad v=0,1,2 \ldots \\
& \hbar \omega_{J}=2 a_{J} \sqrt{B_{J} D_{J}}, \quad \hbar \omega_{J} x_{J}=a_{J}^{2} B_{J}, \quad B_{J}=\hbar^{2}\left(2 m r_{J}^{2}\right)^{-1}, \\
& \psi_{v J}=y^{\eta_{v J}} \exp (-y / 2)_{1} F_{1}(b, c ; y), \\
& y=2 a_{J}^{-1} \sqrt{D_{J} B_{J}^{-1}} \exp \left[-a_{J}\left(r-r_{J}\right) / r_{J}\right], \quad \eta_{v J}=\sqrt{E_{v J} B_{J}^{-1}} \\
& c=2 \eta_{v J}+1, \quad b=c / 2-a^{-1} \sqrt{D_{J} B_{J}}
\end{aligned}
$$


where ${ }_{1} F_{1}(b, c ; y)$ denotes the confluent hypergeometric (or Kummer) function. Introducing relations (11) and (12) into (17), the eigenvalues (16) may be given in an equivalent form

$$
\begin{aligned}
E_{v J} & =\frac{\hbar \omega(v+1 / 2)}{\sqrt{1+B D_{0}^{-1} J(J+1) c_{2}}}-\hbar \omega x(v+1 / 2)^{2}-\frac{\alpha(v+1 / 2) J(J+1)}{\sqrt{1+B D_{0}^{-1} J(J+1) c_{2}}} \\
& +\frac{B J(J+1)}{1+B D_{0}^{-1} J(J+1) c_{2}}-\frac{D J^{2}(J+1)^{2}}{1+B D_{0}^{-1} J(J+1) c_{2}} \\
\hbar \omega x & =a^{2} B, \quad \alpha=B^{3 / 2} D_{0}^{-1 / 2} a c_{1}, \quad D=B^{2} D_{0}^{-1}\left(a^{-1}-2 a^{-2}\right) .
\end{aligned}
$$

A detailed analysis of the obtained results indicates that:

(i) The eigenvalues (21) are given in the form equivalent to that considered by Pekeris [13] and Flügge [12], who applied, however, a different methodology in their derivation.

(ii) The final energy formula takes into account the centrifugal distortion effect which leads to the modification of the potential parameters $D_{0}, a, r_{0}$ and appearance of the rotational terms occurring in (21) in the continued fraction form [14].

(iii) The previous point suggests the possibility of some modification of the energy eigenvalues (21), by replacing the original dissociation constant $D_{0}$ with its multiparametric expansion [8-10]

$$
D_{0} \rightarrow D(J)=D_{0}\left[1+x_{1} J(J+1) /\left[1+x_{2} J(J+1) /[1+\ldots],\right.\right.
$$

where $\left\{x_{i} ; i=1,2 \ldots\right\}$ is the set of external semi-empirical parameters to be obtained by the fitting procedure.

It is interesting to note that the formula (21) is usually considered in the expanded polynomial form of the Dunham type $[12,13]$, for the reason that it permits to relate in the simple manner the spectral Dunham coefficients and the Morse potential parameters. However, in this paper we propose to employ the eigenvalues (21) in the compact form for straightforward evaluation of the potential parameters $\left\{q_{0}, D_{0}, a\right\}$, and next for reproduction of the rovibrational spectra of the selected diatomic systems. The obtained results are collected in Table I which also gives the standard deviation $\sigma$ of the fit, as well as the molecular constants $\{\omega, \omega x, \alpha, B, D\}$ calculated by using the relations (22). The uncertainty in parentheses is one standard deviation in units of the last quoted digit of the fitted parameters. The best values for the molecular parameters are derived by the least-square routine in which the statistical weights proportional to the inverse of experimental uncertainties are taken as being equal to one.

The modified formula (21) including $D(J)$ in the form of a continued fraction (23) is applied to evaluate the molecular parameters $\left\{q_{0}, D_{0}, a, x_{1}, x_{2}\right\}$, and next to reproduce of the rovibrational spectra of the ${ }^{7} \mathrm{LiH}$ molecule.

The rovibrational transitions are calculated from (i) the original eigenvalues (21), (ii) the modified formula (21) obtained by the replacement $D_{0} \rightarrow D(J)$, and from (iii) the polynomial Dunham expansion [15] containing a comparable number of fitted parameters. The results of the calculation are presented in Table II. 
TABLE I

Ground state molecular constants $\left(\mathrm{cm}^{-1}\right)$ for the selected diatomic molecules fitted to the experimental frequencies ${ }^{a}$.

\begin{tabular}{l|l|l|l|l|l|l}
\hline \hline & $\sigma\left(\mathrm{cm}^{-1}\right)$ & $N^{b}$ & $r_{\text {Exp }}^{c}(\AA)$ & $r_{0}(\AA)$ & $D_{0} \times 10^{-3}$ & $a^{d}$ \\
\hline${ }^{12} \mathrm{C}^{34} \mathrm{~S}$ & 0.0009 & 20 & 1.534941 & $1.534781(24)$ & $60.353(21)$ & $2.88967(48)$ \\
${ }^{13} \mathrm{C}^{18} \mathrm{O}$ & 0.0020 & 40 & 1.128323 & $1.1283190(41)$ & $83.837(15)$ & $2.69805(26)$ \\
$\mathrm{H}^{81} \mathrm{Br}$ & 0.0071 & 12 & 1.414435 & $1.41402(12)$ & $35.4409(56)$ & $2.44142(11)$ \\
$\mathrm{DF}$ & 0.0105 & 13 & 0.91694 & $0.917377(66)$ & $41.779(17)$ & $2.22412(35)$ \\
$\mathrm{D}^{37} \mathrm{Cl}$ & 0.0116 & 17 & 1.274581 & $1.27480(14)$ & $38.238(30)$ & $2.35726(74)$ \\
${ }^{115} \mathrm{InD}$ & 0.0025 & 11 & 1.8373 & $1.837641(94)$ & $18.9574(68)$ & $2.40723(34)$ \\
${ }^{132} \mathrm{XeH}+$ & 0.0029 & 16 & $1.602813(6)$ & $1.602695(39)$ & $28.4091(36)$ & $2.63891(12)$ \\
\hline & $\omega$ & $\omega x$ & $\alpha$ & $B$ & $D$ \\
\hline${ }^{12} \mathrm{C}^{34} \mathrm{~S}$ & 1275.5064 & 6.7391 & 0.00567 & 0.807063 & 0.1150 \\
${ }^{a}$ & $1262.0277(4)$ & & & & \\
${ }^{13} \mathrm{C}^{18} \mathrm{O}$ & 2069.2284 & 12.7679 & 0.01425 & 1.753964 & 0.3519 \\
${ }^{e}$ & $2043.69218(20)$ & & & $1.7464072(17)$ & $5.0409(57)$ \\
$\mathrm{H}^{81} \mathrm{Br}$ & 2675.2478 & 50.4851 & 0.20196 & 8.469881 & 14.9906 \\
$c$ & 2648.479 & 45.2175 & 0.23328 & 8.464884 & 34.575 \\
$\mathrm{DF}$ & 3015.5103 & 54.4122 & 0.23243 & 10.999655 & 13.1208 \\
$c$ & 2998.192 & 45.761 & 0.3017 & 11.0102 & 59.4 \\
$\mathrm{D}^{37} \mathrm{Cl}$ & 2148.4326 & 30.1778 & 0.09420 & 5.430916 & 4.9593 \\
$a$ & $2088.073(7)$ & & & $5.3757(3)$ & $13.59(10)$ \\
${ }^{115} \mathrm{InD}$ & 1052.7058 & 14.6142 & 0.04390 & 2.521965 & 5.7898 \\
$c$ & 1048.24 & 12.37 & 0.051 & 2.523 & 5.8 \\
${ }^{132} \mathrm{XeH}+$ & 2278.7109 & 45.6941 & 0.17220 & 6.561665 & 13.9046 \\
$c$ & $2269.9674(11)$ & $41.32056(34)$ & $0.186686(14)$ & $6.560686(14)$ & 21.907 \\
\hline
\end{tabular}

${ }^{a}$ Experimental data: ${ }^{12} \mathrm{C}^{34} \mathrm{~S}(R(J), P(J), v=0 \rightarrow 1)[16],{ }^{13} \mathrm{C}^{18} \mathrm{O}(R(J), P(J), v=$ $0 \rightarrow 1)$ [17], $\mathrm{H}^{81} \mathrm{Br}(R(J), v=1 \rightarrow 3)$ [18], DF $(R(J), v=0 \rightarrow 1)[19], \mathrm{D}^{37} \mathrm{Cl}$ $(R(J), v=0 \rightarrow 1)[20],{ }^{115} \mathrm{InD}(R(J), v=0 \rightarrow 1)[21],{ }^{132} \mathrm{XeH}^{+}(R(J), v=0 \rightarrow 1)$ [22].

${ }^{b}$ Number of the experimental data in the fit.

c Experimental data from Ref. [23], and for ${ }^{132} \mathrm{XeH}^{+}$from Ref. [22].

${ }^{d}$ Parameter dimensionless.

e Experimental data from Ref. [25].

\section{Discussion}

The application of the deformable body model to molecules described by the Morse potential has lead to the fraction continued formula for the rovibrational energy, which seems to be a more general and stronger physically supported equation describing rovibrational states of diatomic systems than the formulae obtained on the basis of the harmonic or Simons-Parr-Finlan potentials. The application 
TABLE II

Ground state molecular constants $\left(\mathrm{cm}^{-1}\right)$ for ${ }^{7} \mathrm{LiH}$ molecule, fitted to 36 experimental frequencies ${ }^{a}$ for $R(J)$ and $P(J)$ band $v=0 \rightarrow 1$.

\begin{tabular}{|c|c|c|c|c|c|c|}
\hline$N^{b}$ & $\sigma\left(\mathrm{cm}^{-1}\right)$ & $r_{0}(\AA)$ & $D_{0} \times 10^{-3}$ & $a^{c}$ & $x_{1}^{c} \times 10^{5}$ & $x_{2}^{c} \times 10^{5}$ \\
\hline 3 & 0.0435 & $1.596205(42)$ & $18.6793(56)$ & $1.88675(34)$ & & \\
\hline 4 & 0.0128 & $1.595829(24)$ & $19.980(73)$ & $1.8190(36)$ & $-2.17(12)$ & \\
\hline 5 & 0.0036 & $1.5958256(66)$ & $19.948(20)$ & $1.8206(10)$ & $-2.167(32)$ & $8.52(47)$ \\
\hline \multirow[t]{2}{*}{ exp. data ${ }^{d}$} & & 1.5957 & & & & \\
\hline & $\sigma\left(\mathrm{cm}^{-1}\right)$ & $\omega$ & $\omega x$ & $\alpha$ & $\bar{B}$ & $D \times 10^{4}$ \\
\hline 3 & 0.0435 & 1413.1452 & 26.7271 & 0.1234 & 7.5080 & 0.9608 \\
\hline 4 & 0.0128 & 1409.4070 & 24.8539 & 0.1022 & 7.5115 & 1.5447 \\
\hline 5 & 0.0036 & 1409.5034 & 24.8984 & 0.1027 & $7: 5116$ & 1.5307 \\
\hline $5^{e}$ & 0.0986 & $1359.595(26)$ & & $0.20864(20)$ & $7.5027(30)$ & $7.84(15)$ \\
\hline $5^{f}$ & 0.0509 & $1359.675(16)$ & & $0.21111(28)$ & $7.5041(10)$ & $8.012(24)$ \\
\hline exp. data ${ }^{d}$ & & 1405.65 & 23.20 & 0.2132 & 7.5131 & 8.617 \\
\hline
\end{tabular}

${ }^{a}$ Experimental data from Ref. [24].

${ }^{b}$ Number of fitted parameters.

cParameter dimensionless.

${ }^{d}$ Experimental data from Ref. [23].

${ }^{e}$ Parameters calculated from 5-parametric Dunham formula $E_{v J}=\omega(v+1 / 2)+[B-\alpha(v+$ $\left.1 / 2)-D J(J+1)+H J^{2}(J+1)^{2}\right] J(J+1)$ with $H=4.1(23) \times 10^{-8}\left(\mathrm{~cm}^{-1}\right)$.

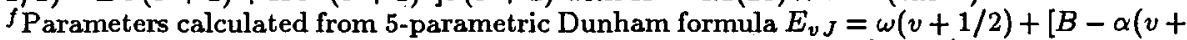
$1 / 2)-[D-\beta(v+1 / 2)] J(J+1)] J(J+1)$ with $\beta=9.52(96) \times 10^{-6}\left(\mathrm{~cm}^{-1}\right)$.

of the Morse function permits to include the rotational dependence of molecular constants $\{\omega, \alpha, B, D\}$ and the anharmonic corrections, so the considered formula may be applied for investigation of the rotational structure of overtone transitions in the highly excited rotational states. Because the equation describing effective rotational energy takes the form of a continued fraction, it permits us to do a straightforward modification of the original eigenvalues using the expansion of the dissociation constant into a continued fraction in the rotational quantum number. Both original and modified formulae provide quite satisfactory relationship between the theoretical and the experimental data over a wide range of rotational states. In particular, the modified formula containing only two semi-empirical parameters $x_{1}$ and $x_{2}$ reproduces the rovibrational transitions of the ${ }^{7} \mathrm{LiH}$ molecule more precisely than the corresponding 5-parametric Dunham expansions. Application of the other 4- or 5-parametric sets of fitted parameters (especially including anharmonic term $\omega x$ ) has led to the worse accuracy of the fit, or even to divergent series.

Inspection of Table II reveals that parameter $x_{1}$ is negative for ${ }^{7} \mathrm{LiH}$ molecule, hence the dissociation constant diminishes with rotational excitation, and the interpretation of $x_{1}$-parameter as an indicator of molecular susceptibility to rotationally induced dissociation also holds true in this case.

Needles to mention that the presented approach allows us to obtain the analytic wave function for the original as well as for the modified eigenvalues, 
therefore, the matrix elements of quantum-mechanical operators, the Franck-Condon factors and intensities of rovibrational transitions can be directly calculated.

\section{Acknowledgment}

I wish to thank Professor J. Konarski for his assistance in writing this paper.

\section{References}

[1] M. Molski, Acta Phys. Pol. A 76, 877 (1989).

[2] M. Molski, Acta Phys. Pol. A 81, 485 (1992).

[3] M. Molski, J. Mol. Struct. 275, 13 (1992).

[4] M. Molski, J. Konarski, Theor. Chim. Acta (1993) (in press).

[5] M. Molski, J. Konarski, Chem. Phys. Lett. 196, 517 (1992).

[6] J. Konarski, J. Mol. Spetrosc. 124, 218 (1987).

[7] J. Konarski, Acta Phys. Pol. A 74, 235 (1988).

[8] M. Molski, J. Konarski, Acta Phys. Pol. A 81, 495 (1992).

[9] M. Molski, J. Konarski, Phys. Rev. A 47, 711 (1993).

[10] M. Molski, J. Konarski, Acta Phys. Pol. A 82, 927 (1992).

[11] P.M. Morse, Phys. Rev. 34, 57 (1927).

[12] S. Flügge, Practical Quantum Mechanics, Springer, Berlin 1974, p. 196 (Rusian edition).

[13] C.L. Pekeris, Phys. Rev. 45, 98 (1934).

[14] W.E. Naniłow, A.N. Iwanowa, J.K. Isakowa, L.A. Lusternik, G.S. Salechow, A.N. Chowanski, L.J. Claf, A.R. Janpolski, Funkcje, granice, szeregi, ulamki lańcuchowe PWN, Warszawa 1970, p: 248 (in Polish).

[15] J.L. Dunham, Phys. Rev. 41, 713 (1932).

[16] C. Yamada, E. Hirota, J. Mol. Spectrosc. 74, 203 (1979).

[17] D. Chen, K.N. Rao, R.S. McDowell, J. Mol. Spectrosc. 61, 71 (1976).

[18] D:H. Rank, U. Fink, T.A. Wiggins, J. Mol. Spectrosc. 18, 170 (1965).

[19] U.K. Sengupta, P.K. Das, K. Narahari Rao, J. Mol. Spectrosc. 74, 322 (1979).

[20] D.U. Webb, K. Narahari Rao, J. Mol. Spectrosc. 28, 121 (1968).

[21] R.D. Urban, H. Birk, P. Polomsky, H. Jones, J. Chem. Phys. 94, 2523 (1991).

[22] S.A. Rogers, C.R. Brazier, P.F. Bernath, J. Chem. Phys. 87, 159 (1987).

[23] K.P. Huber, G. Herzberg, Constants of Diatomic Molecules, Van Nostrand Reinhold, New York 1979.

[24] A.G. Maki, W.B. Olson, G. Thompson, J. Mol. Spectrosc. 144, 257 (1990).

[25] G. Guelachvili, J. Mol. Spectrosc. 75, 251 (1979). 